\title{
Balanced Dissipative Controllers for Flexible Structures
}

\author{
W. Gawronski \\ California Institute of Technology \\ Jerzy T. Sawicki \\ Cleveland State University, j.sawicki@csuohio.edu
}

Follow this and additional works at: https://engagedscholarship.csuohio.edu/enme_facpub

Part of the Mechanical Engineering Commons

How does access to this work benefit you? Let us know!

\section{Original Citation}

Gawronski, W., and Sawicki, J.T.(1997) Balanced Dissipative Controllers for Flexible Structures. Journal of Dynamic Systems, Measurement and Control, 119(1), 5-10, doi: 10.1115/1.2801215.

This Article is brought to you for free and open access by the Mechanical Engineering Department at EngagedScholarship@CSU. It has been accepted for inclusion in Mechanical Engineering Faculty Publications by an authorized administrator of EngagedScholarship@CSU. For more information, please contact library.es@csuohio.edu. 


\section{W. K. Gawronski \\ Member of Technical Staff, \\ Jet Propulsion Laboratory, \\ California Institute of Technology, \\ Pasadena, CA 91109 \\ Balanced Dissipative Controllers for Flexible Structures}

J. T. Sawicki

Assistant Professor,

Cleveland State University,

Cleveland, $\mathrm{OH} 44115$

A balanced approach to shaping the closed-loop properties of the dissipative controllers for flexible structures is presented. In the balanced representation the properties of flexible structures are introduced, and a simple method of designing of the dissipative controllers is obtained. It relates the controller gains with the closed-loop pole locations. The examples illustrate the accuracy of the design method.

\section{Introduction}

Controllers for flexible structures are developed using the root-locus methods (Junkins and Kim, 1993; Meirovitch, 1990), the dissipative properties of the system, see Joshi, (1989) McLaren and Slater (1987) Slater et al. (1992), LQG approach, by Joshi (1989), Junkins and Kim (1993), Meirovitch (1990), Gawronski (1994), and the $H_{\infty}$ approach, by Lim, et al. (1992), Lim and Balas (1992), Gawronski and Lim (1994). The most straightforward approach to the design of controllers for flexible structures is to implement a direct proportional gain between the input and the output. This approach seldom gives superior performance in a general case, since the performance enhancement often causes the reduction of the stability margin. However, if some conditions are satisfied, one obtains a special type of proportional controllers-dissipative ones. The dissipative controllers are often implemented since, as mentioned by Joshi (1989), p. 45, "the stability of dissipative controllers is guaranteed regardless of the number of modes controlled (or even modeled), and regardless of parameter errors." However, the simplicity of the control law does not simplify the design. Typically, in order to obtain required performance typically a large set of inputs and outputs have to be implemented. Determining the gains for this set is not an obvious task. In this paper, we will investigate the properties of the dissipative controllers, and will design of dissipative controllers for flexible structures to meet performance objectives.

\section{Flexible Structure}

For the purposes of this paper, a flexible structure shall be defined as a finite-dimensional, controllable, and observable linear system with small damping and with separated complex poles. A flexible structure is typically represented by the secondorder matrix differential equation

$$
M \ddot{q}+D \dot{q}+K q=B_{o} u, \quad y=C_{o q} q+C_{o v} \dot{q}
$$

In this equation $q$ is the $n_{2} \times 1$ displacement vector, $u$ is the $s$ $\times 1$ input vector, $y$ is the $r \times 1$, the mass $M$ is the $n_{2} \times n_{2}$ matrix, damping $D$ is the $n_{2} \times n_{2}$ matrix, stiffness $K$ is the $n_{2}$ $\times n_{2}$ matrix, the input matrix $B_{o}$ is $n_{2} \times s$, the output displacement matrix $C_{q}$ is $r \times n_{2}$, and the output velocity matrix $C_{v}$ is $r \times n_{2}$. The number $n_{2}$ is the number of degrees of freedom of the system, $r$ is a number of outputs, and $s$ is a number of inputs. The mass matrix is typically positive definite, and the stiffness and damping matrices are typically positive semidefinite.

Define the state vector $x^{T}=\left[q^{T} \dot{q}^{T}\right]$, in which the first component is the system displacement, and the second component is

Contributed by the Dynamic Systems and Control Division for publication in the Journal of DyNamic Systems. MEAsurement, AND Control. Manuscript received by the DSCD December, 1994. Associate Technical Editor: R. C. Redfield. the system velocity. In this case, after elementary manipulations, one obtains the following state-space representation

$$
\begin{gathered}
A=\left[\begin{array}{cc}
0 & I \\
-M^{-1} K & -M^{-1} D
\end{array}\right], \\
B=\left[\begin{array}{c}
0 \\
M^{-1} B_{o}
\end{array}\right], \quad C=\left[\begin{array}{ll}
C_{q} & C_{v}
\end{array}\right]
\end{gathered}
$$

where $A$ is $n \times n, B$ is $n \times s$, and $C$ is $r \times n$. The dimension of the state model $n$ is twice the number of degrees of freedom of the system $n_{2}$, i.e., $n=2 n_{2}$.

The modal state-space representation is further considered. It has a triple $(A, B, C)$ characterized by the block-diagonal matrix $A$,

$$
\begin{aligned}
& A=\operatorname{diag}\left(A_{i}\right), \quad B^{T}=\left[B_{1}^{T}, B_{2}^{T}, \ldots B_{m}^{T}\right], \\
& C=\left[C_{1}, C_{2}, \ldots, C_{m}\right], \quad i=1,2, \ldots, n_{2}
\end{aligned}
$$

where $A_{i}, B_{i}$, and $C_{i}$ are $2 \times 2,2 \times s$, and $r \times 2$ blocks, respectively. For small damping the blocks $A_{i}$ are in the following form

$$
A_{i}=\left[\begin{array}{cc}
-\zeta_{i} \omega_{i} & \omega_{i} \\
-\omega_{i} & -\zeta_{i} \omega_{i}
\end{array}\right]
$$

where $\omega_{i}$ and $\zeta_{i}$ are $i$ th natural frequency and damping. The $i$ th state component, $x_{i}$, corresponding to the $i$ th block has the form $x_{i}=\left[q_{m i} \zeta_{i} q_{m i}+\dot{q}_{m i} / \omega_{i}\right]$, where $q_{m i}$, and $\dot{q}_{m i}$ are $i$ th modal displacement and velocity, as defined in Eq. (4).

The controllability $\left(W_{c}\right)$ and observability $\left(W_{o}\right)$ grammians are used in the design of the dissipative controllers. They are obtained as solutions of the following Lyapunov equations

$$
A W_{c}+W_{c} A^{T}+B B^{T}=0, \quad A^{T} W_{o}+W_{o} A+C^{T} C=0
$$

For stable $A$ the solutions are positive definite.

The system triple is balanced, if its controllability and observability grammians are equal and diagonal, Moore (1981)

$$
\begin{gathered}
W_{c}=W_{o}=\Gamma^{2}, \quad \Gamma=\operatorname{diag}\left(\gamma_{1}, \ldots, \gamma_{n}\right), \\
\gamma_{i} \geq 0, \quad i=1, \ldots, n
\end{gathered}
$$

where $\gamma_{i}$ is the $i$ th Hankel singular value of the system.

The controllability and observability properties of flexible structures are analyzed in Skelton (1980), Gregory (1984), Jonckheere (1984), Williams (1990), Gawronski and Juang (1990), Gawronski and Williams (1991). Assuming small damping, such that $\zeta \ll 1\left(\zeta=\max \left(\zeta_{i}\right), i=1, \ldots, n_{2}\right)$, and separated natural frequencies the matrix $A$ of a balanced flexible structures diagonally dominant 


$$
A \cong \operatorname{diag}\left(A_{i}\right), \quad i=1,2, \ldots, n_{2}
$$

and $A_{i}$ is the modal block as in Eq. (4).

\section{Dissipative Controllers}

The dissipative controllers are based on the Popov's theory of hyperstability, Popov (1973), which was subsequently developed as a positive real property of the control systems Anderson (1967), Benhabib et al. (1987), and as the dissipative (passive) property of the systems Willems (1972), Willems (1976), Desoer and Vidyasagar (1975). The terms: dissipative, passive, positive real, and hyperstable systems are synonyms in our applications, and their inter-relations are discussed by Wen (1988).

Consider a square stable plant $(A, B, C)$, i.e., a linear system with the number of inputs equal to the number of outputs. An open-loop square system with simple poles is dissipative, see Anderson (1967), if there exist a symmetric positive definite matrix $P$ and a matrix $Q$ that satisfy the following equation

$$
A^{T} P+P A=-Q^{T} Q, \quad B^{T} P=C
$$

The system is strictly dissipative if $Q^{T} Q$ is positive definite. This definition allows for simple determination of a dissipative system. For given $A$ and $B$, the matrix $Q$ is selected. Next, one solves the first of Eq. ( 8 ) for $P$, and the output matrix $C$ is found from the second Eq. (8).

The guaranteed stability of the closed-loop system is the most useful property of the dissipative system. It was shown by Desoer (1975), and by Benhabib et al. (1981), that for the square and strictly dissipative plant and the square and dissipative controller (or vice versa: the square and dissipative plant and the square and strictly dissipative controller), the closedloop system is asymptotically stable. In particular, if the feedback gain matrix is a positive definite matrix, the closed-loop system is asymptotically stable.

As a corollary, consider a dissipative system with the statespace representation $(A, B, C)$ which has collocated sensors and actuators, that is, $C=B^{T}$. In this case a closed-loop system with the feedback gain

$$
u=-K y, \text { where } K=\operatorname{diag}\left(k_{i}\right), \quad k_{i}>0,
$$

is stable. This particularly useful configuration can be used only if there is the freedom to choose the collocated sensors and/or actuators, and if the number of the available sensors and actuators is large enough to satisfy the performance requirements. Also, we took advantage of the weakly coupled balanced modes when choosing the diagonal form of the controller gain matrix $K$. There is no need for the off diagonal terms if the modes are independent, or almost independent.

The important class of the dissipative controllers are the lowauthority ones, i.e., such that "allow to modify only moderately the natural modes and frequencies of structures" see Aubrun (1980) and Aubrun and Margulies (1982). Indeed, this kind of controllers is the one used for vibration suppression, Voth et al. (1994), where a moderate effort is needed to suppress the flexible deformations. This kind of controllers is considered later in this paper.

\section{Balanced Dissipative Controllers}

Consider a flexible structure $(A, B, C)$ with an equal number of sensors and actuators. The collocation of sensors and actuator and positive definite gain makes it dissipative. However, flexible structures inherit a property which restricts the collocation of sensors and actuators. The restriction follows from the statespace representation of a flexible structure, as in Eq. (2). In these representations the upper half of the matrix $B$ is equal to zero. Thus, in order to satisfy the collocation requirement, the left half of $C$ must be equal to zero. But the displacement measurements are located in this part. Consequently, a flexible structure is dissipative if the force inputs and the rate outputs are collocated.

In order to determine the properties of the dissipative balanced structures consider further the dissipativity conditions (8) for a flexible structure in the balanced coordinates. Consider also a feedback as in Eq. (9), and let the number of inputs and outputs be $p$. In this case the closed-loop equations are as follows

$$
\dot{x}=(A-B K C) x+B K u_{o}, \quad y=C x
$$

where $u_{o}$ is a control command ( $u_{o}=0$ in the case of vibration suppression ).

We consider further low-authority controllers. Denote $b_{2 i}$ the $i$ th row of $B$, and let $B_{o}$ be a matrix consisting of the diagonal terms of $B B^{T}$, i.e., $B_{o}=\operatorname{diag}\left(b_{i} b_{i}^{T}\right)$. Let $A_{c}$ be the closed-loop matrix, $A_{c}=A-B K B^{T}$. The dissipative controller is of low authority, if $\|K\|=\max \left(k_{i}\right)<k_{o}$, where $k_{o}$ is such that for the closed-loop matrix $A_{c}$ one obtains eig $\left(A_{c}\right) \cong \operatorname{eig}\left(A-{ }^{6} B_{o} K\right)$.

For the low authority dissipative controller the following relationship between $A, B$, and $C$ is valid. For a controllable and observable flexible system there exists a positive constant $k_{o}$ $>0$, such that the dissipative controller is of low authority. Furthermore, if $A$ is in the modal form one can use the following equations that relate $A, B$, and $C$

$$
\begin{aligned}
B B^{T} & \cong C^{T} C \cong-\Gamma^{2}\left(A+A^{T}\right) \\
& =\operatorname{diag}\left(\alpha_{1}, \alpha_{1}, \alpha_{2}, \alpha_{2}, \ldots, \alpha_{n}, \alpha_{n}\right),
\end{aligned}
$$

which, for the $i$ th block, it translates to

$$
B_{i} B_{i}^{T} \cong C_{i}^{T} C_{i} \cong-\gamma_{i}^{2}\left(A_{i}+A_{i}^{T}\right)=\alpha_{i} I_{2}
$$

where $\alpha_{i}=2 \gamma_{i}^{2} \zeta_{i} \omega_{i}, B_{i}$, is the two-row block of $B$, and $C_{i}$ is the two-column block of $C$.

In order to show it note that $b_{i} K b_{j}^{T}$ is the $i j$ th term of $B K B^{T}$. Since $\left(b_{i} K b_{j}^{T}\right)^{2} \leq\left(b_{i} K b_{i}^{T}\right)\left(b_{j} K b_{j}^{T}\right)$, therefore, for $A$ in the modal form (3), and for small gain $K$, such that $\|K\|=\max$ $\left(k_{i}\right)<k_{o}$, the off-diagonal terms of $B K B^{T}$ do not influence the eigenvalues of $A_{c}$, and they can be ignored. Consequently, Eqs. $(11 a, b)$ can be used in the closed loop equations.

Since the matrix $A$ is in the modal form, and $K$ is diagonal, $K=\operatorname{diag}\left(k_{1}, \ldots k_{p}\right)$, then in the balanced coordinates with collocated sensors and actuators one obtains the closed-loop matrix $A_{c}=A-B K B^{T}$ in the form

$$
A_{c}=A-\sum_{j=1}^{p} k_{j} B_{j} B_{j}^{T}
$$

where $B_{j}$ is the $j$ th column of $B$. In the balanced coordinates matrix $A_{c}$ is diagonally dominant, that is, $A_{c} \cong \operatorname{diag}\left(A_{c 1}, \ldots\right.$ $A_{c n}$ ), where $A_{c i}$ is $2 \times 2$ block. For this block the Eq. (12) is as follows

$$
A_{c i} \cong A_{i}-\sum_{i=1}^{p} k_{j} B_{j i} B_{j i}^{T}
$$

In this equation the cross terms $B_{j k} B_{j i}^{T}$ (for $k \neq i$ ) were omitted as negligible in the balanced coordinates, see Eq. (11b), where $B_{j i}$ is the $i$ th block of the $j$ th column of $B$. Also, for the balanced system the following holds $B_{j i} B_{j i}^{T} \cong-\gamma_{j i}^{2}\left(A_{i}+A_{i}^{T}\right)$, see Eq. $(11 b)$, where $\gamma_{j i}$ is the $i$ th Hankel singular value obtained for the $j$ th column of $B$, i.e., for the triplet $\left(A, B_{j}, B_{j}^{T}\right)$. Thus, Eq. (12) is now

$$
A_{c i} \cong A_{i}+2 \sum_{j=1}^{p} k_{j} \gamma_{j i}^{2}\left(A_{i}+A_{i}^{T}\right),
$$

and introducing $A_{i}$ as in Eq. (4), and noting that $A_{i}+A_{i}^{T}=$ $-2 \zeta_{i} \omega_{i} I_{2}$, as in Eq. $(11 b)$, one rewrites Eq. (13) 


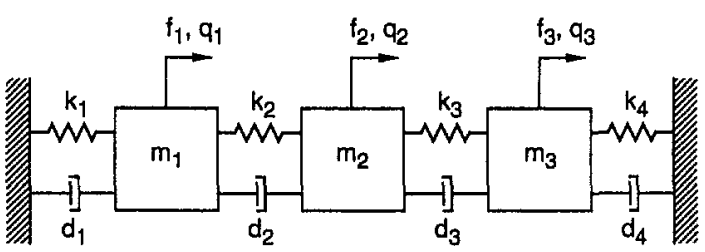

Fig. 1 A simple system

$$
A_{c i} \cong\left[\begin{array}{cc}
-\beta_{i} \zeta_{i} \omega_{i} & -\omega_{i} \\
\omega_{i} & -\beta_{i} \zeta_{i} \omega_{i}
\end{array}\right]
$$

with the parameter $\beta_{i}$ given as

$$
\beta_{i}=1+2 \sum_{j=1}^{p} k_{j} \gamma_{j i}^{2}
$$

Comparing the closed-loop matrix as in Eq. (14a) and the openloop matrix as in Eq. (4) it can be seen that $\beta_{i}$ is a measure of the shift of the $i$ th pair of poles. Denote the closed-loop pair of poles $\left(\lambda_{c r i}, \pm j \lambda_{c i i}\right)$ and the open-loop pair $\left(\lambda_{\text {ori }}, \pm j \lambda_{\text {oii }}\right)$, then it follows from Eq. (14) that they are related

$$
\left(\lambda_{c r i}, \pm j \lambda_{c i i}\right) \cong\left(\beta_{i} \lambda_{o r i}, \pm j \lambda_{o i i}\right), \quad i=1, \ldots, n
$$

where $\beta_{i}$ is defined in Eq. (14b). Equation (15) shows that the real part of the $i$ th pair of poles is shifted, while the imaginary part is stationary. The shift is proportional to the gain of each input, and to the $i$ th Hankel singular values associated with each input.

Equation (14) sets the basic limitation for the dissipative controller design: that the number of inputs (and outputs) limits the number of controlled modes (or controlled pairs of poles). In order to illustrate it, assume a single-input-single-output system. In this case $\beta_{1 i}=1+2 k_{1} \gamma_{1 i}^{2}$, and the scalar gain $k_{1}$ is the only free parameter available for the design. Thus only one pole can be shifted to the required position. If more than one pair should be shifted, their placement would be a least-square compromise, which typically would be non-satisfactory. Thus, in order to avoid this rough approximation, it is often required for the dissipative controllers to have a large number of sensors and actuators to meet the required performance criteria.

The pole-shift coefficient $\beta_{i}$ can be interpreted as a ratio of the open $\left(\gamma_{o i}\right)$ - and closed-loop $\left(\gamma_{c i}\right)$ Hankel singular values, or as a ratio of the variances of open-loop $\left(\sigma_{o i}^{2}\right)$ and closedloop $\left(\sigma_{c i}^{2}\right)$ states excited by the white noise input, i.e.,

$$
\beta_{i} \cong \frac{\gamma_{o i}^{2}}{\gamma_{c i}^{2}}=\frac{\sigma_{o i}^{2}}{\sigma_{c i}^{2}}
$$

This interpretation follows from the closed-loop Lyapunov equation

$$
\left(A-B K B^{T}\right) \Gamma_{c}^{2}+\Gamma_{c}^{2}\left(A-B K B^{T}\right)^{T}+B B^{T}=0
$$

which for the $i$ th pair of variables is as follows

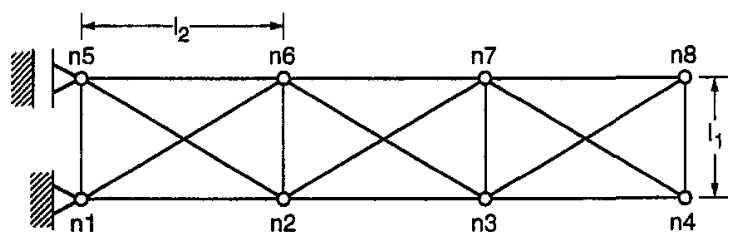

Fig. 2 A truss structure

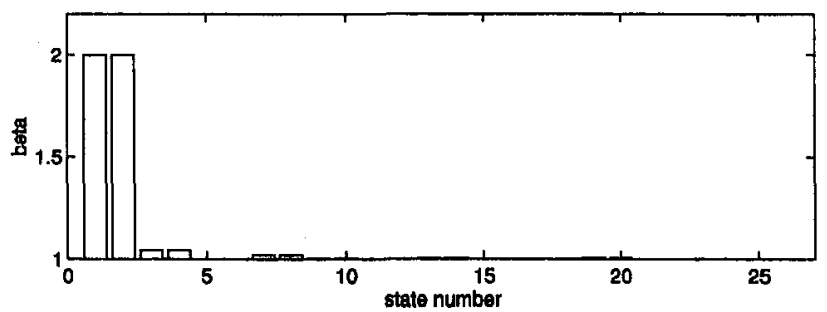

Fig. 3 Coefficient $\beta$, for the single-input-single-output truss

$\left(A_{i}-\sum_{j=1}^{p} k_{i} B_{j i} B_{j i}^{T}\right) \gamma_{c i}^{2}$

$$
+\gamma_{c i}^{2}\left(A_{i}-\sum_{j=1}^{p} k_{i} B_{j i} B_{j i}^{T}\right)^{T}+B_{i} B_{i}^{T} \cong 0
$$

Introducing Eq. ( $11 b)$, after some algebra, one obtains

$$
\gamma_{c i}^{2}+2 \gamma_{c i}^{2}\left(\sum_{j=1}^{p} k_{j} \gamma_{j i}^{2}\right)-\gamma_{o i}^{2} \cong 0
$$

or, finally

$$
\frac{\gamma_{o i}^{2}}{\gamma_{c i}^{2}} \cong 1+2 \sum_{j=1}^{p} k_{j} \gamma_{j i}^{2}=\beta_{i}
$$

Based on Eqs. (14b), (15), and (16) a tool for pole placement of the dissipative controllers is developed. The task is to determine gains $k_{j}, j=1, \ldots, p$, such that the selected poles are placed at the required location (or as close as possible in the least-square sense). Equivalently, the task is to determine gains $k_{j}, j=1, \ldots, p$, such that the input noise of the selected modes is suppressed at ratio $\beta_{i}$. The approach follows from Eq. $(14 b)$, since one can determine the gains such that $q$ poles are shifted by $\beta_{i}, i=1, \ldots, q$, i.e., $\lambda_{c r i}=\beta_{i} \lambda_{o r i}$, or the noise be suppressed by $\beta_{i}$, i.e., $\sigma_{o i}^{2}=\beta_{i} \sigma_{c i}^{2}$. Define the gain vector $k$

$$
k=\left[k_{1}, k_{2}, \ldots, k_{p}\right]^{T}
$$

so that Eq. (14b) can be re-written as

$$
d \beta \cong G k
$$

where $d \beta$ is the vector of pole shifts

$$
d \beta=\left[\beta_{1}-1, \beta_{2}-1, \ldots, \beta_{q}-1\right]^{T}
$$

and $G$ is the matrix of the system Hankel singular values for each actuator and sensor location

$$
G=2\left[\begin{array}{cccc}
\gamma_{11}^{2} & \gamma_{21}^{2} & \cdots & \gamma_{p 1}^{2} \\
\gamma_{12}^{2} & \gamma_{22}^{2} & \cdots & \gamma_{p 2}^{2} \\
\cdots & \cdots & \cdots & \cdots \\
\gamma_{1 q}^{2} & \gamma_{2 q}^{2} & \cdots & \gamma_{p q}^{2}
\end{array}\right]
$$

where $\gamma_{i j}$ is the $j$ th Hankel singular value for the $i$ th actuator/ sensor location.

The least-square solution of Eq. (21) is obtained

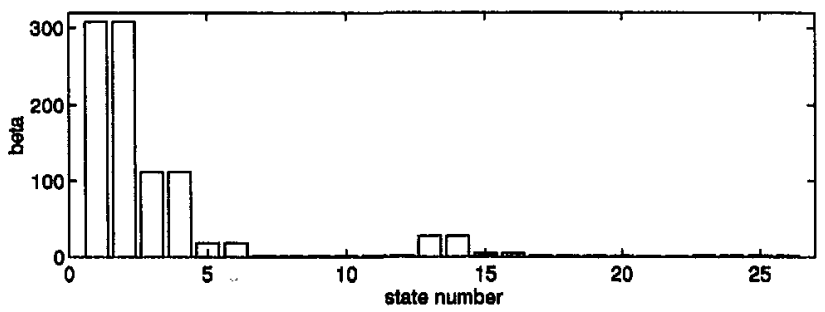

Fig. 4 Coefficient $\beta_{1}$, for the two-input-two-output truss 


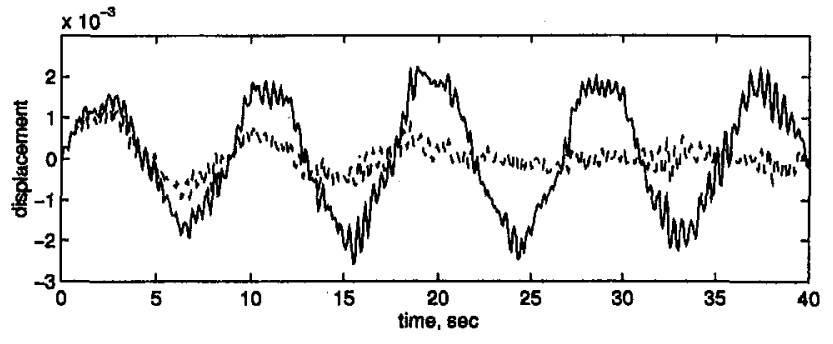

Fig. 5 Open- and closed-loop output due to disturbances

$$
k \cong G^{+} d \beta
$$

where $G^{+}$is pseudoinverse of $G$. The set of Eqs. (21) is either overdetermined $(q>p$, or rank $(G)=p$ ), or square $(q=p$ $=\operatorname{rank}(G))$, or underdetermined $(q<p$, or $\operatorname{rank}(G)=q)$, see Golub and Van Loan (1989). The form of pseudoinverse depends on the number of inputs and outputs $p$, and the number of poles shifted, $q$, that is, on the rank of the matrix $G$.

\section{Design Examples}

The examples of balanced dissipative controller design are presented for the simple flexible system, and for the truss structure.

Simple System. The system is shown in Fig. 1, with the masses $m_{1}=m_{2}=m_{3}=I$, stiffness $k_{1}=10, k_{2}=k_{4}=3, k_{3}$ $=4$, and the damping matrix $D$ as a linear combination of the mass $(M)$ and stiffness $(K)$ matrices, $D=0.004 K+0.001 M$. The input force is applied to the mass $m_{3}$, the output is the rate of the same mass. The poles of the open-loop system are

$$
\begin{gathered}
\lambda_{o 1, o 2}=-0.0024 \pm j 0.9851, \quad \lambda_{o 3, o 4}=-0.0175 \pm j 2.9197, \\
\lambda_{o 5, o 6}=-0.0295 \pm j 3.8084 .
\end{gathered}
$$

The system open-loop balanced representation was obtained with the following vector of Hankel singular values

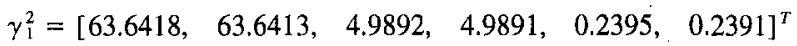

It is required to shift the first pole by increasing its damping twofold, and leave the other poles stationary. For this shift coefficients are $\beta_{1}=2$, and $\beta_{2}=\beta_{3}=1$, therefore $d \beta=$ $\left[\begin{array}{llllll}1 & 1 & 0 & 0 & 0 & 0\end{array}\right]^{T}$. Also for this case $G=2 \gamma_{1}^{2}$, thus the gain $k=0.0078$ is obtained from Eq. (23). For this gain, the closed-loop eigenvalues were computed

$$
\begin{gathered}
\lambda_{c 1, c 2}=-0.0049 \pm j 0.9851, \quad \lambda_{c 3, c 4}=-0.0189 \pm j 2.9197, \\
\lambda_{c 5, c 6}=-0.0296 \pm j 3.8084
\end{gathered}
$$

and from this result one can see that the actual shifts $\beta_{1}=$ $1.9939, \beta_{2}=1.0779$, and $\beta_{3}=1.0037$ are close to the required ones.

Next, consider a design which increases the first and the second pole damping twofold, and leaves the third stationary. In this case $\beta_{1}=\beta_{2}=2$, and $\beta_{3}=1$ is required, therefore $d \beta$ $=\left[\begin{array}{llllll}1 & 1 & 1 & 1 & 0 & 0\end{array}\right]^{T}$. The gain $k=0.0084$ is obtained from Eq. (23), and the closed-loop eigenvalues for this gain are computed:

$$
\begin{gathered}
\lambda_{c 1, c 2}=-0.0051 \pm j 0.9851, \quad \lambda_{c 3, c 4}=-0.0190 \pm j 2.9197, \\
\lambda_{c 5, c 6}=-0.0296 \pm j 3.8084 .
\end{gathered}
$$

Comparing the open- and closed-loop poles, one can see that the actual shifts, $\beta_{1}=2.0718, \beta_{2}=1.0840$, and $\beta_{3}=1.0040$, are almost the same as in the first case (small shift in the second pole is observed). Thus, we hardly met the requirements. This case shows that for the underdetermined problem (number of inputs is smaller than the number of poles to be shifted), the obtained least square result is not satisfactory, although it is the best one can get in the given situation.

Truss Structure. The truss is presented in Fig. 2. A single control force is applied at node $n 7$, directed vertically. The output is a rate collocated with the force. The system has 26 balanced states. The most controllable and observable mode is suppressed by increasing the damping of this mode two times. The required feedback gain, as in Eq. (9), is obtained from Eq. (23). In order to do this, note that in this case $\beta_{1}=2$, and the remaining $\beta$ 's are equal to 1 . Let $\gamma_{1}$ be a vector of the Hankel singular values for the system, then $G=\gamma_{1}$. For this case $d \beta$ $=\left[\begin{array}{llllllll}2 & 2 & 1 & 1 & 0 & 0 & \ldots & 0\end{array}\right]$, and one obtains the gain $k=1548.5$ from Eq. (23). For this gain the closed-loop poles were determined, and the pole shift was obtained as a ratio of real parts of closed- and open-loop poles, as in definition Eq. (15), i.e., $\beta_{i}=\lambda_{c r i} / \lambda_{\text {ori }}$. The plot of $\beta_{i}$ is shown in Fig. 3. It shows that $\beta_{1}=1.9978 \cong 2$, as required. The damping of the first pole increased two times, while the other poles changed insignificantly.

Next, two control forces are applied at node $n 7$. The first one is directed vertically, the second one, horizontally. The outputs are the collocated rates. The disturbance force $d$ at node $n 8$, at $y$-direction, was added. The suppression of vibrations due to disturbances is required. It is done by introducing pole shift of 300 for the first mode, and of 100 for the second mode, obtaining $d \beta=[299,299,99,99,0, \ldots 0]$. For this case, one obtains the gains $k=[3.127,2.688] 10^{5}$ from Eq. (23). The shift for the first mode was 307 , and for the second mode 111 (see Fig. 4), i.e., close to the required one. The open- and the closed-loop system response to the white noise disturbance $d$ is shown in Fig. 5, where the improved disturbance rejection property is observed.

\section{Conclusions}

A simple method, which relates the closed-loop poles with the gains of the balanced dissipative controllers of flexible structures is presented. The effectiveness of the method is illustrated with the controller design examples of a simple flexible system, and the truss structure.

\section{References}

Anderson, B. D. O., 1967, “A System Theory Criterion for Positive Real Matrices," J. SIAM Control, Vol. 5, pp. 171-182.

Aubrun, J. N., 1980, "Theory of the Control of Structures by Low-Authority Controllers," Journal of Guidance, Control, and Dynamics, Vol. 3.

Aubrun, J. N., and Margulies, G., 1982, 'Low-Authority Control Synthesis for Large Space Structures," NASA Contractor Report 3495, Contract NAS114887

Benhabib, R. J., Iwens, R. P., and Jackson, R. L., 1981, "Stability of Large Space Structure Control Systems Using Positivity Concepts," Journal of Guidance and Control, Vol. 4, pp. 487-494.

Desoer, C. A., and Vidyasagar, M., 1975, Feedback Systems: Input-Output Properties, Academic Press, New York.

Gawronski, W., 1994, "A Balanced LQG Compensator for Flexible Structures," Automatica, Vol. 30.

Gawronski, W., and Lim, K. B., 1994, "Balanced $H_{\infty}$ and $H_{2}$ Controllers," Proc. 1994 American Control Conference, Baltimore, MD, pp. 1116-1122.

Gawronski, W., and Juang, J. N., 1990, "Model Reduction for Flexible Structures," Control and Dynamics Systems, C. T. Leondes, ed., Vol. 36, Academic Press, San Diego, CA, pp. 143-222.

Gawronski, W., and Williams, T., 1991, "Model Reduction for Flexible Space Structures," Journal of Guidance, Control, and Dynamics, Vol. 14, pp. $68-76$.

Golub, G. H., Van Loan, C. F., 1989, Matrix Computations, The Johns Hopkins University Press, Baltimore, MD.

Gregory, Jr., C. Z., 1984, "Reduction of Large Flexible Spacecraft Models Using Internal Balancing Theory," J. Guidance, Control and Dynamics, Vol. 7, pp. 725-732.

Jonckheere, A., 1984, "Principal Component Analysis of Flexible SystemsOpen Loop Case," IEEE Trans. Autom. Control, Vol. 27, pp. 1095-1097.

Joshi, S. M., 1989, Control of Large Flexible Space Structures, Springer, Berlin.

Junkins, J. L., and Kim, Y., 1993, Introduction to Dynamics and Control of Flexible Structures, Published by the American Institute of Aeronautics and Astronautics, Washington, D.C. 
Lim, K. B., and Balas, G. J., 1992, "Line-of-Sight Control of the CSI Evolutionary Model: $\mu$ Control," IEEE American Control Conf., Chicago, IL.

Lim, K. B., Maghami, P. G., and Joshi, S. M., 1992, "Comparison of Controller Designs for an Experimental Flexible Structure," IEEE Control Systems Magazine, pp. 108-118.

McLaren, M. D., and Slater, G. L., 1987, "Robust Multivariable Control of Large Space Structures Using Positivity," Journal of Guidance, Control, and Dynamics, Vol. 10, pp. 393-400.

Meirovitch, L., 1990, Dynamics and Control of Structures, Wiley, New York. Moore, B. C., 1981, "Principal Component Analysis in Linear Systems, Controllability, Observability and Model Reduction," IEEE Trans. Autom. Control, Vol. 26, pp. 17-32

Popov, V. M., 1973, Hyperstability of Automatic Control Systems, Springer, New York.

Skelton, R. E. 1980, "Cost Decomposition of Linear Systems with Application to Model Reduction," Int. J. of Control, Vol. 32, pp. 1031-1055.
Slater, G. L., Bosse, A. B., and Zhang, Q., 1992, "Robustness of Positive Real Controllers for Large Space Structures," Journal of Guidance, Control, and Dynamics, Vol. 15, pp. 58-64.

Voth, C. T., Richards Jr., K. E., Schmitz, E., Gehling, R. N., and Morgenthaler, D. R., "Integrated Active and Passive Control Design Methodology for the LaRC CSI Evolutionary Model," NASA Contractor Report 4580, Contract NAS1$19371,1994$.

Wen, J. T., 1988, "Time Domain and Frequency Domain Conditions for Strict Positive Realness," IEEE Trans. on Automat. Control, Vol. AC-33, p. 988-992. Willems, J. C., 1972, "Dissipative Dynamical Systems, Part I: General Theory, Part II: Linear Systems with Quadratic Supply Rates," Arch. Rational Mech. and Anal. Vol. 45, pp. 321-351, and op. 352-392.

Willems, J. C., 1976, "Realization of Systems with Internal Passivity and Symmetry Constraints," J. Franklin Institute, Vol. 301, pp. 605-620.

Williams, T., 1990, "Closed Form Grammians and Model Reduction for Flexible Space Structures," IEEE Trans. on Automatic Control, Vol. AC-35, pp. 379-382. 